\title{
Evaluación del efecto sobre el metabolismo hídrico de la ciudad Bogotá como respuesta al cambio en el modelo de gestión del agua en los años 90
}

Evaluation of the Effect on the Water Metabolism due to the Change in the Water Management Model in Bogotá during the 1990s

\author{
Yulia Ivanova ${ }^{a}$ \\ Universidad Militar Nueva Granada, Colombia \\ yulia.ivanova@unimilitar.edu.co \\ ORCID: http://orcid.org/0000-0001-8836-5175 \\ Efrain Antonio Dominguez Calle \\ Pontificia Universidad Javeriana, Colombia \\ ORCID: http://orcid.org/0000-0001-5487-9661 \\ Armando Sarmiento \\ Pontificia Universidad Javeriana, Colombia \\ ORCID: http://orcid.org/0000-0002-1561-3622
}

DOI: https://doi.org/10.11144/Javeriana.ayd22-42.eesm Redalyc: http://www.redalyc.org/articulo.oa? id $=151557418001$

Fecha de recepción: 15 Septiembre 2017 Fecha de publicación: 30 Junio 2018

\section{Resumen:}

Durante la decada de los noventa, Colombia y, en particular, la ciudad de Bogotá ha experimentado cambios en sus modelos de la gestión del recurso hídrico. En este artículo se calculó la huella hídrica de Bogotá para evaluar la incidencia de los cambios de modelo de gestión sobre el consumo del líquido y los vertimientos de aguas en la capital de la república. Como resultado, se obtuvo que la ciudad, entre 1993 y 2008, logró disminuir sus consumos en un $40 \%$ a pesar de su progresivo crecimiento y de que el decrecimiento de la presión sobre el recurso hídrico es una respuesta a los cambios administrativos, legislativos y culturales adelantados en Bogotá durante el periodo de tiempo mencionado. No obstante, el tema de manejo de vertimientos resultó ser un eslabón crítico, ya que la huella hídrica gris representa el 55,8\% de la huella total de la capital, en la cual los aportes de vertimientos en la cuenca del río Tunjuelo resaltan como el mayor contaminante.

Palabras clave: ciudad de Bogotá, consumos del agua, contaminación del recurso hídrico, gestión del agua en áreas urbanas, huella hídrica.

\begin{abstract}
:
During the 1990s, Colombia -and particularly, the city of Bogotá- underwent some changes in the models of water resource management. This paper provides a calculation of the water footprint in Bogotá in order to evaluate how the changes in the water management models impacted the water consumption and the wastewater discharges in the city of Bogotá. As a result, it was found that between 1993 and 2008 the city managed to decrease the consumption numbers in 40\%, despite the gradual city growth and thanks to the fact that the pressure on the water resources decreased due to the administrative, legislative, and cultural changes implemented in Bogotá during the abovementioned time. However, the issue of wastewater discharge treatment showed to be a critical link, as the gray water footprint represents $55.8 \%$ of the total water footprint in the city. The wastewater discharges poured into the Tunjuelito River catchment area are the higher pollutant.
\end{abstract}

Keywords: Bogotá, water consumption, water resource pollution, urban area water management, water footprint.

\section{Introducción}

En la modernidad, el tema de la gestión del agua en Colombia ha estado influenciado por las dinámicas ocurridas en la América Latina en los años 90. En esta época, se sintió una profunda crisis en las instituciones estatales gestoras del agua, lo que demuestró su insuficiencia económica, la cual, en conjunto con los elevados consumos del recurso hídrico, en consonancia con tarifas bajas y subsidios otorgados por el Estado, incentivó el uso irracional del agua en los sectores consumidores. Con el fin de enfrentar esta situación, la mayoría de los países de América Latina han cambiado su modelo de la gestión del recurso 
hídrico de lo estatal hacia la gestión privada, con elementos de control estatal y participación ciudadana. Colombia también siguió éstas dinámicas (Const., 1991), sustentando el nuevo modelo de la gestión del agua con el nuevo aparato legislativo y organizacional (Empresa de Acueducto de Bogotá [EAAB], 2003). Inicialmente, para responder a la necesidad de la reestructuración del sector del agua, se promulgó la Ley 142 de 1994, que abrió la posibilidad de la participación de las empresas privadas en la gestión del recurso hídrico. Como ente de control de regulación del servicio del agua y de la fijación de las tarifas, se creó la Comisión de Regulación de Agua Potable y Saneamiento Básico (Ley 142, 1994, art. 69). Con el fin de vigilar e inspeccionar los servicios públicos, bajo la Ley 142 de 1994 se consolida la Superintendencia de los Servicios Públicos Domiciliarios que se adscribe al Ministerio de Desarrollo Económico (Artículo 76). $\mathrm{Al}$ estar en concordancia con uno de los principios fundamentales de la Constitución Nacional de 1991 (Const., 1991, art. 2), se creó el Comité de Desarrollo y Control Social (Congreso de la República, 1994).

Para crear un sistema autofinanciado de las empresas prestadoras de los servicios de acueducto y alcantarillado, se crearon los conceptos bajo los cuales opera el nuevo modelo de la gestión del recurso hídrico: eficiencia económica, neutralidad, solidaridad, redistribución, suficiencia financiera, simplicidad y transparencia (Ley 142, 1994) El sector legislativo respondió a la reestructuración de la institucionalidad, introduciendo cambios en el sistema del cobro por el uso del agua, las tasas retributivas y las propuestas en la definición de los límites máximos permisibles de los vertimientos a los cuerpos del agua.

Por un lado, respecto a la tasa por uso del agua, se introdujo el concepto de la estratificación socioeconómica. Según esta división, los estratos económicos cinco y seis contribuyen al pago de las tarifas de los estratos uno, dos y tres, mientras que el estrato cuatro paga la tarifa del agua, correspondiente a su costo real. De esa forma, según las fuentes de información, el estrato uno paga tan solo el $50 \%$ del costo real de la tarifa.

Por otro, para controlar los vertimientos se aplica el cobro de la tasa retributiva. Según esta, se pretende disminuir los niveles de contaminación hasta que sean socialmente aceptables, internalizando las externalidades negativas de los agentes económicos (Correa-Restrepo, Ossa-Arteaga, \& Vallejo-Chanci, 2007). En términos económicos, esta situación se denomina eficiencia sin optimabilidad, cuando no se pretende eliminar la contaminación, sino disminuirla hasta un nivel que sea socialmente adecuado (Galarza-García \& Rudas-Lleras, 2009).

En el tema de la definición de los límites máximos permisibles de los vertimientos, con el Decreto 1594 de 1984 se reglamentaron los usos del agua y residuos líquidos. Al tener en cuenta la permisibilidad de la norma, por la definición de los límites de vertimientos en función de la remoción de algunos parámetros de calidad de aguas y no por su valor límite permisible, se han generado algunas contradicciones en materia ambiental: Algunos artículos de este Decreto fueron derogados por la Sentencia del 14 de agosto de 1994. Tan solo en el 2010, fue presentada, por el Ministerio de Medio Ambiente y Desarrollo Sostenible (MADS), una propuesta de la nueva norma para los parámetros máximos permisibles en función del agente contaminante que se concluyó en la Resolución 631 de 2015 . El efecto de esta norma sobre la calidad del río Bogotá aún no puede ser evaluada por la disponibilidad de los datos.

Conjunto con todos los cambios normativos y legislativos, la política cívica de Antanas Mockus ha influenciado sobre el ahorro voluntario de agua por parte de la población citadina, durante las época de los años 90 (Serrano, 2016).

Todos los factores mencionados anteriormente, de una u otra forma han tenido un impacto en la relación entre la sociedad capitalina y el agua. Para medir las consecuencias de esta relación, en el articulo se aplicó la metodología desarrollada por Arjen Hoekstra (Hoekstra, Chapagain, Aldaya, \& Mekonnen, 2011), con elfi $\mathrm{n}$ de identificar los volúmenes directos e indirectos del agua que sustentan la vida y el desarrollo de la capital. El período de análisis comprende entre 1993 y 2008, dictado para identificar la incidencia de los cambios institucionales y legislativos sobre los consumos per cápita del agua y los efectos de la contaminación de la cuenca media del río Bogotá, considerando la disponibilidad de la información para este fin. 


\section{Materiales y Métodos}

La relación de las ciudades con el agua es compleja y está en constante dinámica bajo los cambios normativos, legislativos, culturales y tecnológicos (Mekonnen, Pahlow, Aldaya, Zarate, \& Hoekstra, 2015) . Es posible evaluar esta evolución mediante la metodología de la huella hídrica, formulada por Arjen Hoekstra en los años 90 (Hoekstra, 2015). El término de huella hídrica fue propuesto como indicador de presión sobre el recurso hídrico. El término se entiende cómo "el uso de agua que tiene en cuenta tanto el uso directo como indirecto por parte de un consumidor o productor" (Hoekstra et al., 2011).

Por su parte, la huella se divide en tres tipos, en función del origen de las aguas usadas para satisfacer las demandas del recurso (Bosire et al., 2017). Así, la huella hídrica azul se relaciona con el uso de las aguas superficiales y subterráneas, la huella hídrica verde se refiere al uso de las aguas provenientes de las lluvias (Zhuo, Mekonnen, \& Hoekstra, 2016) , y la huella hídrica gris hace referencia al volumen del agua requerido para neutralizar la concentración de los contaminantes introducidos a uno u otro cuerpo hídrico (Hoekstra et al., 2011). Teniendo en cuenta que las aguas lluvias no se emplean explícitamente por los sectores consumidores del agua, esta no fue considerada en este estudio como componente de la huella hídrica verde (Mekonnen \& Hoekstra, 2010).

Para el caso de la ciudad de Bogotá, la huella hídrica azul se compone de dos elementos. El primero son los consumos facturados de agua en el período considerado, que se llamarán consumos directos; el segundo se relaciona con los volúmenes anuales del líquido, calculados a partir del Índice del agua no contabilizada (IANC), que representa el agua suministrada a la red del acueducto, pero que no fue consumida por los suscriptores y sus dependientes por causas como fugas, conexiones ilícitas, suministro del agua para las demandas en el sector social, etc. Este índice no se relaciona directamente con los consumos del agua, pero sí se debe tener en cuenta a la hora de calcular la huella hídrica azul, ya que estos volúmenes de agua corresponden a las especificaciones técnicas de la red del acueducto, y representan la afectación de las corrientes hídricas por la extracción del recurso. En relación con lo anterior, la huella hídrica azul se calcula de la siguiente forma:

$$
\mathrm{HH}_{\text {azul }}=\mathrm{HH}_{\text {consumos directos }}+\mathrm{HH}_{\text {LANC }} \quad \text { Ecuación } 1
$$

En la cual:

$\mathrm{HH}_{\text {azul }}$ - Huella hídrica azul de la ciudad, $\left(\mathrm{m}^{3}\right)$

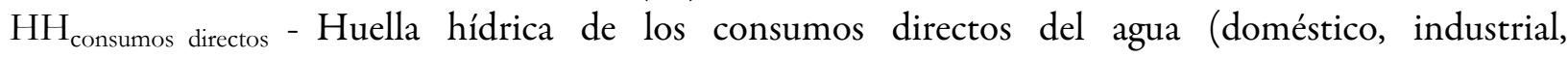
comercial y otros, comprendidos en el período de 1993 a $2008,\left(\mathrm{~m}^{3}\right)$

$\mathrm{HH}_{\mathrm{IANC}}$-Volúmenes anuales del agua, correspondientes al índice del agua no contabilizada, $\left(\mathrm{m}^{3}\right)$

La segunda etapa en la aplicación de la metodología consiste en la estimación de la huella hídrica gris, que son los volúmenes de agua dulce requeridos para asimilar la carga contaminante por la corriente hídrica en la cual están vertidos dichos contaminantes. Teniendo en cuenta que la descarga de los vertimientos de la ciudad al río Bogotá se realiza en tres puntos, la huella hídrica gris se compone de los tres elementos correspondientes a las descargas de los vertimientos después de la Planta de Tratamiento de Aguas Residuales (PTAR) El Salitre, y a los efluentes de los ríos Fucha y Tunjuelo. Considerando las dificultades relacionadas con la disponibilidad de la información histórica de los vertimientos al río Bogotá, como los volúmenes de las descargas y su concentración de los SST (sólidos totales en suspensión), y la concentración de los SST a lo largo del cauce del río Bogotá, la huella hídrica gris se calculó para el año 2008, para el cual hubo disponible toda la información requerida. En la tabla 1 se presentan los volúmenes de los vertimientos y su caracterización por SST para cada uno de los puntos de los vertimientos al cauce del río Bogotá, que es uno de los componentes para la estimación de la huella hídrica gris de la ciudad. 
TABLA 1.

Caudal de descarga y la concentración de sólidos totales en suspensión

(SST) de los tres vertimientos de la ciudad al río Bogotá (año 2008)

\begin{tabular}{lcc}
\hline Cuenca & $\boldsymbol{Q},(\boldsymbol{l} / \boldsymbol{s})$ & $\boldsymbol{S S T},\left(\frac{\boldsymbol{m g}}{\boldsymbol{l}}\right)$ \\
\hline Salitre & 819,3 & 55,0 \\
Fucha & 1991,4 & 70,0 \\
Tunjuelito & 9803,0 & 232,0 \\
\hline
\end{tabular}

Fuente: Superintendencia de Servicios Públicos (SSP), 2018

La estimación de la huella hídrica gris usa los valores de los sólidos totales en suspensión antes de los vertimientos, y el de los vertimientos. Su propósito es contrastar cómo las descargas de los vertimientos se diferencian de los valores naturales de los parámetros de calidad del río. Los sitios de monitoreo de calidad de aguas, correspondientes a los puntos ubicados aguas arriba de los vertimientos de la ciudad de Bogotá, son los puntos relacionados con Lisboa, Aguas Arriba de la Estación Gibraltar y San Bernandino. La estación Lisboa corresponde al punto antes de los vertimientos realizados por la PTAR El Salitre. La estación Aguas Arriba de la Estación Gibraltar es punto de monitoreo de la calidad de aguas 100 metros antes del vertimiento de la cuenca del río Fucha, y el punto de monitoreo denominado San Bernandino corresponde a la ubicación antes de los vertimientos del río Tunjuelo. La ubicación de estos puntos y los parámetros de sus vertimientos se presentan en la tabla 2.

TABLA 2.

Puntos de seguimiento de la calidad del recurso hídrico antes de los vertimientos realizados por las cuencas Salitre, Fucha y Tunjuelito

\begin{tabular}{llll}
\hline Nombre del punto de & \multicolumn{2}{l}{ Coordenadas } & $\begin{array}{l}\text { Concentraciones de los SST } \\
\text { monitoreo }\end{array}$ \\
\cline { 2 - 3 } & Longitud & Latitud & $\begin{array}{l}\text { (mg/l) en la cuenca media } \\
\text { del río Bogotá }\end{array}$ \\
\hline Lisboa & 4,44224 & 74,07505 & 23 \\
100 metros aguas arriba de & 4,38566 & 74,10555 & 85 \\
la descarga de la estación & & & \\
Gibraltar & & & \\
San Bernandino & 4,37438 & 74,13067 & 61 \\
\hline
\end{tabular}

Fuente: Secretaría Distrital de Ambiente (SDA) y Empresa de Acueducto de Bogotá (EAAB), 2008

La huella hídrica gris correspondiente a la cuenca del río Salitre se expresa a través de la siguiente ecuación:

$$
H H_{\text {gris Salitre }}=\frac{L_{\text {Salitre }}}{C_{\text {máx }}-C_{\text {Lisboa }}} \quad \text { Ecuación } 2
$$

En la cual: 
$\mathrm{L}_{\text {Salitre }}$ - Descarga del contaminante a través de la PTAR Salitre, $(\mathrm{kg} / \mathrm{s})$

$\mathrm{C}_{\text {máx }}$ - Concentración máxima permisible de los SST, $\left(\mathrm{kg} / \mathrm{m}^{3}\right)$. El valor máximo permisible del vertimiento puntual de aguas residuales domésticas y no domésticas a cuerpos de aguas continentales de los SST es de $200 \mathrm{mg} / \mathrm{L}$ para las instalaciones existentes del sistema del alcantarillado (MADS, 2015).

$\mathrm{C}_{\text {Lisboa }}$ - Concentración de los SST medida por la estación de calidad de aguas Lisboa, $\left(\mathrm{kg} / \mathrm{m}^{3}\right)$.

Para el cálculo de la huella hídrica gris de los vertimientos generados por el Fucha se tuvieron en cuenta los siguientes componentes: $L$ - Descarga de los SST vertidos directamente al río Bogotá a través del río Fucha (masa/tiempo); $\mathrm{C}_{100 \mathrm{~m}}$ antes de Gibraltar - concentración de los SST antes de los vertimientos generados a través del río Fucha. El punto de monitoreo de calidad de aguas se encuentra en cercanía del vertimiento del río Fucha y se encuentra 100 metros aguas arriba de la descarga de la estación Gibraltar.

$$
H_{\text {gris.Fuc ha }}=\frac{L_{\text {Fucha }}}{C_{\max }-C_{100 \text { mantes de Gibraltar }}} \quad \text { Ecuación } 3
$$

Para el cálculo de la huella hídrica gris de los vertimientos generados por el Fucha se tuvieron en cuenta los siguientes componentes: - Concentración del contaminante vertido directamente al río Bogotá a través del río Tunjuelo (masa/tiempo); - concentración de los SST antes de los vertimientos generados por el río Tunjuelo. El punto de monitoreo se encuentra en cercanía del vertimiento y se denomina "San Bernandino". Con base en lo mencionado anteriormente, la huella hídrica para la cuenca del río Tunjuelo se calcula a través de la siguiente ecuación:

$$
H H_{\text {gris.Tunjuelito }}=\frac{L_{\text {Tunjuelo }}}{C_{\max }-C_{\text {nat.San Bernandino }}} \quad \text { Ecuación } 4
$$

Los valores de la huella hídrica gris se convirtieron a datos anuales para su posterior inclusión a la ecuación general de la huella hídrica de Bogotá.

La huella hídrica total de la ciudad de Bogotá se calculó como la sumatoria de las huellas hídricas gris y azul, correspondientes al año 2008.

$$
H H_{\text {total }}=H H_{a z u l}+H H_{g r i s} \quad \text { Ecuación } 5
$$

En la cual:

$\mathrm{HH}_{\text {total }}$ - Huella hídrica total, $\left(\mathrm{m}^{3} /\right.$ año $)$

$\mathrm{HH}_{\text {azul }}$ - Huella hídrica azul, $\left(\mathrm{m}^{3} /\right.$ año $)$

$\mathrm{HH}_{\text {gris }}$ - Huella hídrica gris, $\left(\mathrm{m}^{3} /\right.$ año $)$

\section{Resultados y discusión}

Huella hídrica azul: como se había mencionado anteriormente, el concepto de la huella hídrica azul se relaciona con la identificación de los volúmenes superficiales del agua que se requieren, tanto para satisfacción de las necesidades básicas de la, como para el desarrollo de las actividades productivas. En la tabla 3 se presenta la evolución histórica de los consumos del agua en la ciudad en el período 1993 al 2008. 
TABLA 3.

Evolución histórica de los consumos del agua en la ciudad de Bogotá

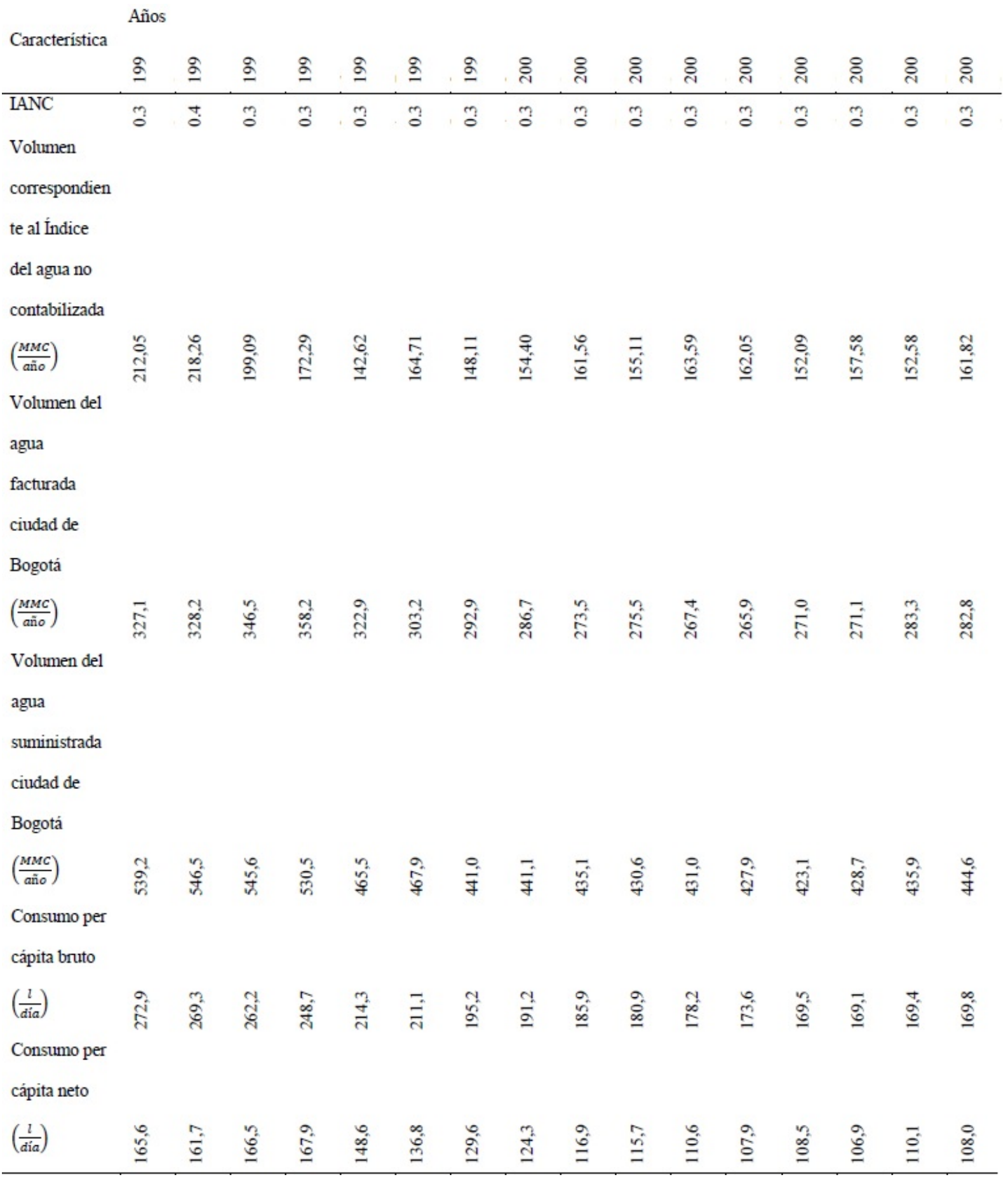

Fuente: (SSP, 2018)

Como se puede evidenciar en la tabla 3, en los últimos 15 años los consumos del agua en la capital han disminuido considerablemente, al pasar de 539,3 a 444,6 millones de metros cúbicos al año. En general, el consumo bruto ha disminuido en un $17,6 \%$, considerando el crecimiento progresivo poblacional de la ciudad.

Si los consumos de agua no se hubieran afectado en las últimas décadas por los cambios institucionales, tarifarios y simbólicos, teniendo en cuenta la población al año 2008, los consumos anuales de agua serían cercanos a los 712,6 millones de metros cúbicos. Bogotá, sin haber implementado todas las reformas 
mencionadas anteriormente, hubiera teniendo, para el 2008, unos consumos de agua alrededor del $40 \%$ mayores a los que en realidad se regitraron durante este año. Este hecho demuestra cómo los cambios legislativos, tecnológicos y tarifarios influenciaron positivamente en los patrones de uso del agua en la capital. Al tener en cuenta que los consumos del líquido se dividen en los facturados y suministrados, para cada uno de estos se pueden analizar las dinámicas y factores que han afectado su evolución. Los volúmenes del agua facturada se relacionan con los volúmenes directamente consumidos por los usuarios. La disminución de dichos consumos en el período considerado se debe a un abanico de factores que no se pueden considerar de forma segregada porque estaban influenciando conjuntamente en el mismo período histórico. Entre estos se pueden relacionar los cambios en el sistema tarifario, la política de cultura ciudadana de Antanas Mockus (Arbeláez y Rivadulla, 2010) y la gestión de crisis del derrumbre de uno de los túneles del sistema de abastecimiento de Chingaza en el año 1997, hecho que generó los racionamientos de agua más significativos de la ciudad.

En la tabla 3 se puede apreciar cómo después de 1997 comenzó a disminuir el volumen de agua facturada en la ciudad, posiblemente por la concientización de la población en la necesidad del uso racional del recurso hídrico. Como consecuencia de todos estos factores, el consumo per cápita neto en la capital colombiana pasó de 165,5 litros/día en 1993, a 108,0 litros/día en el 2008, valor que concuerda con la Resolución 330 de 2017, (Ministerio de Vivienda, Ciudad y Territorio, 2017) en la cual se recomiendan 120 litros/persona/ día para la dotación neta máxima de las poblaciones localizadas en las alturas mayores de 2000 metros sobre el nivel del mar.

En relación a los volúmenes del agua no contabilizada, en los primeros años del periodo analizado el índice tuvo valores más altos, y después de 1997 comenzó a disminuir gracias al programa de micromedición puesto en marcha por la EAAB y al Programa de Control de Pérdidas adelantado después de 1997. Es así como, entre 1993 y 2008, los valores del índice del agua no contabilizada disminuyeron de, aproximadamente, 39\% hasta un $36 \%$, lo que representa un volumen del agua de 56,4 millones de metros cúbicos al año. En los últimos años, el IANC se encuentra relativamente constante, lo que se justifica por las capacidades técnicas de controlar pérdidas y la justificación de costo/beneficio de la reducción de dichas pérdidas.

Huella hídrica gris: como se mencionó en el apartado anterior, en el trabajo realizado se evaluó la huella hídrica gris correspondiente al 2008. Este hecho se explica por las limitaciones relacionadas con la disponibilidad de la información histórica relacionada con los parámetros de los vertimientos. En la tabla 4 se presentan los resultados correspondientes a la huella hídrica gris para la ciudad de Bogotá. Según lo mencionado en la tabla, la huella hídrica gris anual de la capital se aproxima a los 562, 242 . El mayor porcentaje de dicha huella corresponde a la cuenca del río Tunjuelo, cuyos volúmenes de agua requeridos para la descontaminación de los vertimientos equivalen al 91,8\% de la huella hídrica gris de la ciudad. El hecho de que el mayor aporte a esta pertenezca al río Tunjuelo, se debe a las actividades socioeconómicas desarrolladas en esta cuenca hidrográfica y a la pobre infraestructura sanitaria troncal y secundaria, por lo cual su cauce recibe casi la totalidad de los vertimientos de los barrios en su vertiente sur. Según algunas estimaciones realizadas, a la contaminación del río Tunjuelo aportan en relación porcentual el 78,05\% el sector de las curtiembres, el $8,78 \%$ el sector de los alimentos y el 5,37\% la industria textil (SDA y EAAB, 2008). El porcentaje restante pertenece a los sectores químico, metalmecánico, estaciones de servicio, entre otros. Adicionalmente, aproximadamente el 34,6\% de la población citadina reside en esta cuenca hidrográfica, y vierten sus aguas residuales directamente al río Tunjuelo. Los aportes del río Fucha son segundos por su importancia dada las concentraciones de los sólidos totales en suspensión que se vierten a la cuenca media del río Bogotá (SDA y EAAB, 2008). Las cargas contaminantes se dan principalmente por el sector de alimentos, talleres metalmecánicos, el sector de textiles y la industria química, entre otros (SDA y EAAB, 2008). 
TABLA 4 .

Datos iniciales y los resultados de la huella hídrica gris de la ciudad de Bogotá

\begin{tabular}{lllllll}
\hline Cuenca & C nat, $(\mathrm{mg} / \mathrm{l})$ & C máx, $(\mathrm{mg} / \mathrm{l})$ & Q vert., $(\mathrm{l} / \mathrm{s})$ & C vert., $(\mathrm{mg} / \mathrm{l})$ & $\mathrm{L},(\mathrm{mg} / \mathrm{s})$ & $\mathrm{HH}$ gris $(\mathrm{Mm} / \mathrm{año})$ \\
\hline Salitre & 23,0 & 200,0 & 819,3 & 55,0 & 45061,5 & 8,028 \\
Fucha & 85,0 & 200,0 & 1991,4 & 70,0 & 139398,0 & 38,23 \\
Tunjuelo & 61,0 & 200,0 & 9803,0 & 232,0 & 2274296,0 & 515,98 \\
\multicolumn{2}{l}{ Huella hídrica total: } & & & & & 562.242 \\
\hline
\end{tabular}

Fuente: Secretaría Distrital de Ambiente (SDA) y Empresa de Acueducto de Bogotá (EAAB), 2008

A pesar de que la descontaminación de las aguas residuales provenientes de los canales Torca y El Salitre no es completa, la remoción de los SST disminuye el aporte de estas subcuencas hidrográficas a la carga contaminante resultante al río Bogotá. Los sectores que mayores aportes tienen en la concentración de sólidos suspendidos totales son las estaciones de servicios, el sector de alimentos y los hospitales (SDA y EAAB, 2008).

Huella hídrica total: con base en los resultados parciales de las huellas hídricas azul y gris, se calculó la huella hídrica total de la ciudad de Bogotá, correspondiente al 2008, la cual alcanzó el valor de 1,006 millones de metros cúbicos al año. El mayor porcentaje de la huella hídrica total pertenece a la huella hídrica gris. El porcentaje de contribución de la huella hídrica gris al valor total de la huella hídrica de Bogotá es de 55,8\%, el cual equivale a los 444,600 de agua consumidos al año. La huella hídrica gris para Bogotá, estimada con base en los parámetros del 2008, alcanza un valor de 562,242 necesarios para remover la concentración de los SST hasta su concentración natural. Bajo el principio de "responsabilidad de la cuna a la tumba" (MesaCuadros, 2010), las aguas servidas deben ser entregadas en las condiciones que las caracterizaban en la etapa de captación. En este orden de ideas, la huella hídrica gris debería tener valores cercanos a cero, y los valores obtenidos identifican un manejo inadecuado de los vertimientos. Igualmente, el tratamiento parcial de los vertimientos recibidos por la PTAR Salitre (EAAB, 2009) y la ausencia de plantas de tratamiento de aguas residuales sobre los ríos Fucha y Tunjuelo, que son los mayores aportantes de contaminantes, implican que la huella hídrica gris de Bogotá no tenga una evolución positiva hacia la disminución de las tasas contaminantes.

\section{Conclusiones}

Como conclusión general se puede relacionar que el cambio en el modelo de gestión del agua fue abordado desde el punto de vista integral, sumando los efectos de los cambios tecnológicos, culturales, normativos, condiciones que, según (González-Ladrón-de-Guevara, 2006), son necesarios para una solución íntegra de una problemática o necesidad en materia ambiental. Esto ha posibilitado una disminución significativa de los consumos del agua en la ciudad de Bogotá. 
No obstante, la capital no ha tenido el mismo enfoque integral en el abordaje del tema de los vertimientos. El primer obstáculo en su análisis se relaciona con la ausencia de una sola fuente de información confiable, ya que diferentes fuentes presentan valores disparejos de los parámetros de vertimientos (Ballesteros, 2007).

En segundo lugar, no existen registros históricos de los vertimientos de la ciudad para poder evaluar su evolución a través de los años. En tercer lugar, el valor de la huella hídrica gris, estimada con base en los datos de vertimientos del año 2008, afirma que el efecto de los vertimientos supera el efecto de la extracción del recurso hídrico. Este hecho indica notables deficiencias en los sistemas de tratamiento de aguas residuales, prioritariamente en la cuenca del río Tunjuelo, que aporta el mayor porcentaje a la contaminación del río Bogotá en comparación con las otras dos fuentes de vertimiento (ríos Salitre y Fucha). Esto alarma sobre la necesidad de gestionar los vertimientos en la cuenca del río Tunjuelo con el fin de contribuir al mejoramiento de las condiciones ambientales del agua del río Bogotá.

Esta gestión debe ser entendida desde el concepto de los cambios legislativos y tecnológicos, y desde la necesidad de la implementación de los instrumentos económicos para regular las tasas de vertimientos al cuerpo hídrico.

Adicionalmente, se concluye que la metodología de la huella hídrica se puede aplicar no solo para algunos sectores económicos (Lovarelli, Bacenetti, y Fiala, 2016) como el sector agricultor (Duan, Qin, Wang, y He, 2016), el industrial (Carmona, 2015), o el energético (Gu, Dong, y Wang, 2016), sino también como un indicador de presión sobre el recurso hídrico en áreas urbanas (Sun, Lu, y Xie, 2015), posibilitando la comparación de las tasas de uso del agua retributivas con los niveles de contaminación del recurso hídrico, bajo diferentes contextos legislativos, administrativos y culturales

\section{Notas}

Este artículo es producto del trabajo de grado de Yulia Ivanova, presentado en la Maestría en Gestión Ambiental, de la Pontificia Universidad Javeriana de Bogotá.

\section{Referencias}

Arbeláez, N., y Rivadulla, M.J. (2010). Concepción teórica y práctica de la ciudadanía en el proyecto de cultura ciudadana. Estudio de caso: ahorro voluntario de agua. Bogotá D.C.: Universidad Colegio Mayor de Nuestra Señora del Rosario.

Ballesteros, P. (2007). Evaluación de tasas retributivas por vertimientos hidricos en la cuenca media del rio Bogotá. Bogotá: Escuela Superior de Administración Pública.

Bosire, C. K., Lannestad, M., De Leeuw, J., Krol, M. S., Ogutu, J. O., Ochungo, P. A., y Hoekstra, A. Y. (2017). Urban consumption of meat and milk and its green and blur water footprints - Patterns in tre 1980s and 2000s for Nairobi, Kenya. Science if the Total Environment, 786 - 796 . https://doi.org/10.1016/j.scitotenv.2016.11.027

Carmona, L. (2015). Water Footprint for Heavy Oil Extraction in Colombia: Relationship between Oil and Water. Society of petroleum engineers. https://doi.org/10.2118/173552-MS

Congreso de Colombia. (11 de julio de 1994). Por la cual se establece el régimen de los servicios públicos domiciliarios y se dictan otras disposiciones [Ley 142 de 1994]. Recuperado de http://www.secretariasenado.gov.co/senado /basedoc/ley_0142_1994.html

Constitución de Colombia [Const.]. (1991).

Correa-Restrepo, F., Ossa-Arteaga, A., y Vallejo-Chanci, Z. (2007). Regulación ambiental en Colombia: el caso de la tasa retributiva para el control de la contaminación hídrica. Semestre económico, 10(19), 27-46.

Duan, P., Qin, L., Wang, Y., y He, H. (2016). Spatial pattern characteristics of water footprint for maize production in Northearest China. Journal of the Science of food and agriculture, 96(2), 561 - 568. https://doi.org/10.1002/ jsfa.7124 
Empresa de Acueducto de Bogotá (EAAB). (2003). El agua en la historia de Bogotá 1538 - 2003. Bogotá: Villegas editores.

EAAB. (2009). Plegable técnico - PTAR Salitre, fase I. Bogotá: Eaab.

Galarza-García, M. A. y Rudas-Lleras, G. (2009). Análisis de efectividad de las tasas retributivas en Colombia. Bogotá: Pontificia Universidad Javeriana.

González-Ladron-de-Guevara, F. J. (2006). En busca de caminos para la comprensión de la problemática ambiental. La enscisión moderna entre cultura u naturaleza. Bogotá: Pontificia Universidad Javeriana.

$\mathrm{Gu}$, Y., Dong, Y. y Wang, N. (2016). Quantification of the water, energy and carbon wootprints of wastewater taetmen plants in China considering a water energy nexus perspective. Ecologycal Indicators, 60, 402 - 409. https:// doi.org/10.1016/j.ecolind.2015.07.012

Hoekstra, A. Y. (2015). The water footprint: The relation between human consumption and water use. En M. Antonelli y F. Greco (Eds.), The Water We Eat: combining virtual and water footprints (pp. 35 - 48). Switzerland: Springer International Publishing.

Hoekstra, A., Chapagain, A., Aldaya, M., y Mekonnen, M. (2011). The Water Footprint Assessment Manual. Setting the Global Standard. Recuperado de https://waterfootprint.org/media/downloads/TheWaterFootprintAssess mentManual_2.pdf

Lovarelli, D., Bacenetti, J., y Fiala, M. (2016). Water footprint of crop production: A review. Science of the Total Environment, 549-549, 236-251. https://doi.org/10.1016/j.scitotenv.2016.01.022

Ministerio de Ambiente y Desarrollo Sostenible (MADS). (Marzo 17 de 2015). Por la cual se establecen los parámetros y los valores límites máximos permisibles en los vertimientos puntuales a cuerpos de aguas superficiales y a los sistemas de alcantarillado público y se dictan otras disposiciones. [Resolución 631]. Recuperado de https://doc s.supersalud.gov.co/PortalWeb/Juridica/OtraNormativa/R_MADS_0631_2015.pdf

Mekonnen, M. M. y Hoekstra, A. Y. (2010). A global and high - resolution asessment of the green, blue and greywater footprint of wheat. Hydrology and Earth Science, 14, 1259 - 1276. https://doi.org/10.5194/hess-14-1259-2010

Mekonnen, M. M., Pahlow, M., Aldaya, M. M., Zarate, E., y Hoekstra, A. Y. (2015). Sustainability, efficiency and equitability of water consumption and pollution in latin America and the Caribbean. Sustainability (Switzerland), 7(2), 2086 - 2112. https://doi.org/10.3390/su7022086

Mesa-Cuadros, G. (2010). Derechos ambientales en perspectiva de integralidad : concepto y fundamentación de nuevas demandas y resistencias actuales hacia el "estado ambiental de derecho". Bogotá: Universidad Nacional de Colombia.

Secretaría Distrital de Ambiente (SDA) y Empresa de Acueducto de Bogotá (EAAB) (2008). Calidad del sistema hídrico del Bogotá. Recuperado de http://ambientebogota.gov.co/documents/24732/3987336/Calidad+del+s istema+hidrico+de+Bogot\%C3\%A1.pdf

Serrano, M. I. (2016). Cultura ciudadana desde la transmisión: análisis del caso de Anthanas Mockus en Bogotá. Bogotá: Pontificia Universidad Javeriana.

Superintendencia de Servicios Públicos (SSP). (2018). Servicio Único de Información de servicios públicos domiciliarios. Recuperado de http://www.sui.gov.co/web/

Sun, Y., Lu, C., y Xie, G. (2015). Water footprint in Beijing. Chinese Journal of Ecology, 34, 524 - 531.

Zhuo, L., Mekonnen, M. M., y Hoekstra, A. Y. (2016). Inter - and intra -annual variation of water footprint of crops and blue water scarcity in the Yellow River basin (1961 - 2009). Advances in Water Resouces, 87, 29 - 41. https:// doi.org/10.1016/j.advwatres.2015.11.002

\section{Licencia Creative Commons CC BY 4.0}

Cómo citar este artículo: Ivanova, Y., Domínguez, E. A., y Sarmiento, A. (2018). Evaluación del efecto sobre el metabolismo hídrico de la ciudad bogotá como respuesta al cambio en el modelo de gestión del agua en los años 90. Ambiente y Desarrollo, 22(42), 1-10. https://doi.org/10.11144/Javeriana.ayd22-42.eesm 